EPJ manuscript No.

(will be inserted by the editor)

\title{
Forward dispersion relations and Roy equations in $\pi \pi$ scattering
}

\author{
R. Kamiński ${ }^{1}$, J. R. Peláez ${ }^{2}$ and F. J. Ynduráin ${ }^{3}$ \\ 1 Department of Theoretical Physics Henryk Niewodniczański Institute of Nuclear Physics, Polish Academy of Sciences, 31-242, \\ Kraków, Poland \\ 2 Departamento de Física Teórica, II (Métodos Matemáticos), Facultad de Ciencias Físicas, Universidad Complutense de \\ Madrid, E-28040, Madrid, Spain \\ 3 Departamento de Física Teórica, C-XI Universidad Autónoma de Madrid, Canto Blanco, E-28049, Madrid, Spain
}

Received: date / Revised version: date

\begin{abstract}
We review results of an analysis of $\pi \pi$ interactions in $S, P$ and $D$ waves for two-pion effective mass from threshold to about $1.4 \mathrm{GeV}$. In particular we show a recent improvement of this analysis above the $K \bar{K}$ threshold using more data for phase shifts and including the $S 0$ wave inelasticity from $\pi \pi \rightarrow K \bar{K}$. In addition, we have improved the fit to the $f_{2}(1270)$ resonance and used a more flexible $P$ wave parametrization above the $K \bar{K}$ threshold and included an estimation of the $D 2$ wave inelasticity. The better accuracy thus achieved also required a refinement of the Regge analysis above $1.42 \mathrm{GeV}$. We have checked that the $\pi \pi$ scattering amplitudes obtained in this approach satisfy remarkably well forward dispersion relations and Roy's equations.
\end{abstract}

PACS. 1 3.75.Lb

\section{Introduction}

In a previous analysis [1] a set of fits to different data sets on $\pi \pi$ scattering was presented together with a detailed description of the mathematical methods used in calculations. Forward dispersion relations (FDR) were then used , in order to test the correctness of the amplitudes thus constructed. Remarkably, it was found that some of the very frequently used sets of phase shifts do not satisfy FDR below $1 \mathrm{GeV}$. Thus FDR were shown to give strong constraints to fits which, when used later as a constraint, lead to an improved and precise representation of $\pi \pi$ scattering amplitudes below, roughly $1 \mathrm{GeV}$. In the regions from about $1 \mathrm{GeV}$ to $1.4 \mathrm{GeV}$ there was still some mismatch between the real part of the amplitudes and the results of dispersive evaluations in [1] (especially for $\pi^{0} \pi^{0}$ scattering).

In a subsequent article [2], in order to improve the agreement with the constraints given by FDR, we have reanalysed the parametrizations of the $S 0$ above $K K$ threshold, the $D 0$ wave and to a lesser extend the $P$ and $D 2$ waves. In the $S 0$ wave we took into account systematically the elasticity data from the $\pi \pi \rightarrow K \bar{K}$ reaction [3-7], included in the fit more data on phase shifts above the $K \bar{K}$ threshold [3-6] and used more a flexible parametrization from $0.932 \mathrm{GeV}$ to $1.4 \mathrm{GeV}$. In the $D 0$ wave we have used experimental data from $[3,6,8]$, information on low energy parameters (the scattering length and slope) and included in the fit the width and mass of the $f_{2}(1270)$ resonance as given by the PDG [9]. The result is that, for both $S 0$ and
$D 0$ waves, we have obtained more accurate parametrizations with smaller errors compared to those in the previous approach [1]. In the $P$ wave we have exploited a more flexible parametrization between the $K \bar{K}$ threshold and 1.42 $\mathrm{GeV}$ and in the $D 2$ wave we have included its estimated inelasticity above $1 \mathrm{GeV}$.

This more accurate determination of the $\pi \pi$ amplitudes below $1.42 \mathrm{GeV}$ allowed us to refine the Regge analysis that had been used in $[1,10]$. This has been done by removing the degeneracy condition $\alpha_{\rho}(0)=\alpha_{P^{\prime}}(0)$ which thus modifies slightly the central values of the intercepts $\alpha_{\rho}(0)$ (by $\left.\sim 11 \%\right)$ and $\alpha_{P^{\prime}}(0)$ (by $\left.\sim 4 \%\right)$, but yields smaller errors than those in [1].

We have found that the $\pi \pi$ amplitudes with the new parametrizations of phase shifts and inelasticities in the $S, P$ and $D$ waves together with the just discussed small changes in $\alpha_{\rho}(0)$ and $\alpha_{P^{\prime}}(0)$ allow for much better fulfilment of FDR than in [1]. The biggest improve in $\chi^{2}$ (about $66 \%$ ) is achieved for the forward $\pi^{0} \pi^{0}$ dispersion relation and a smaller one (about $15 \%$ ) for $\pi^{0} \pi^{+}$. In the case of the forward dispersion relation for isopin 1 in the $t$-channel a very tiny deterioration has been found ( $\chi^{2}$ increased by about $26 \%$ ), which is still acceptable, since, considering all FDR together, there is a considerable overall improvement in their fulfilment. It is worth noting that this has been achieved despite the improved data fits have smaller errors than in [1].

We have also tested Roy equations, which, contrary to FDR, incorporate s-t crossing, by calculating the difference between the real parts of the input amplitudes and 
those obtained from Roy's equations. We have found that, on average, and up to almost the $K K$ threshold, the deviation from zero is smaller that 1.05 times the value of the errors for the $S 0$ wave, smaller that 1.2 for the $S 2$ wave and smaller than 0.65 for the $P$ wave.

\section{$2 S, P$ and $D$ waves at higher energies but below $1.42 \mathrm{GeV}$}

In this section the main features of the new paramaterizations of $S, P$ and $D$ waves between roughly the $K \bar{K}$ threshold and $1.42 \mathrm{GeV}$ are presented. Details of each parametrization can be found in [2]. Since the description of the $S 2$ wave was not changed in [2], any information on this wave is available in [1].

\subsection{The $S 0$ wave}

In the present approach we obtain both the tangent of the phase shifts $\tan \delta_{0}^{0}$ and the inelasticity $\eta_{0}^{0}$ above $0.932 \mathrm{GeV}$ as functions of $\mathbf{K}$-matrix elements

$$
K_{i j}(s)=\frac{\mu \alpha_{i} \alpha_{j}}{M_{1}^{2}-s}+\frac{\mu \beta_{i} \beta_{j}}{M_{2}^{2}-s}+\frac{1}{\mu} \gamma_{i j},
$$

where $i, j=1,2$ denote $\pi$ or $K$ respectively, and we set the mass scale $\mu=1 \mathrm{GeV}$. All $\alpha_{i}, \beta_{i}$ and $\gamma_{i}$ are determined from the fit. Note that $M_{1}=0.9105 \pm 0.0070$ $\mathrm{GeV}$ simulates the left hand cut of the $\mathbf{K}$-matrix located at $2 \sqrt{M_{K}^{2}-m_{\pi}^{2}}=0.952 \mathrm{GeV}$ and the pole at $M_{2}=$ $1.324 \pm 0.006 \mathrm{GeV}$ is connected with $\delta_{0}^{0}$ passing through $270^{\circ}$. The parametrizations: of [1] (below $0.932 \mathrm{GeV}$ ) and of [2] (above $0.932 \mathrm{GeV}$ ) are matched at $0.932 \mathrm{MeV}$. In the fit all data on phase shifts below and above the $K \bar{K}$ threshold [3-6] have been used simultaneously. For $\eta_{0}^{0}$, data from $\pi \pi \rightarrow K \bar{K}$ have been used together with data on $\pi \pi \rightarrow \pi \pi$ [3-7]. The resulting fit yields $\chi^{2} /$ d.o. $f=0.6$ and can be seen in Fig. 1.

\subsection{The $D 0$ wave}

For this wave we proceeded by fitting simultaneously below and above the $K \bar{K}$ a parametrization

$$
\cot \delta_{2}^{(0)}=\frac{s^{1 / 2}}{2 k^{5}}\left(M_{f_{2}}^{2}-s\right) m_{\pi}^{2}\left(B_{0}+B_{1} w(s)\right)
$$

with $w(s)=\frac{\sqrt{s}-\sqrt{s_{0}-s}}{\sqrt{s}+\sqrt{s_{0}-s}}$, but using different $B_{i}$ and $s_{0}$ parameters above and below $K K$ threshold. We also required both parametrizations to match at $\sqrt{s}=2 m_{K}$, thus eliminating one parameter. In the present approach, the mass of the $f_{2}(1270)$ resonance $M_{f_{2}}$ was fixed to the PDG value [9]. The $B_{i}$ parameters have been obtained for those two energy regions from fits to experimental data points $[3,6$, 8] together with three other constraints: the width of the $f_{2}(1270)$ resonance from [9], plus the scattering length and
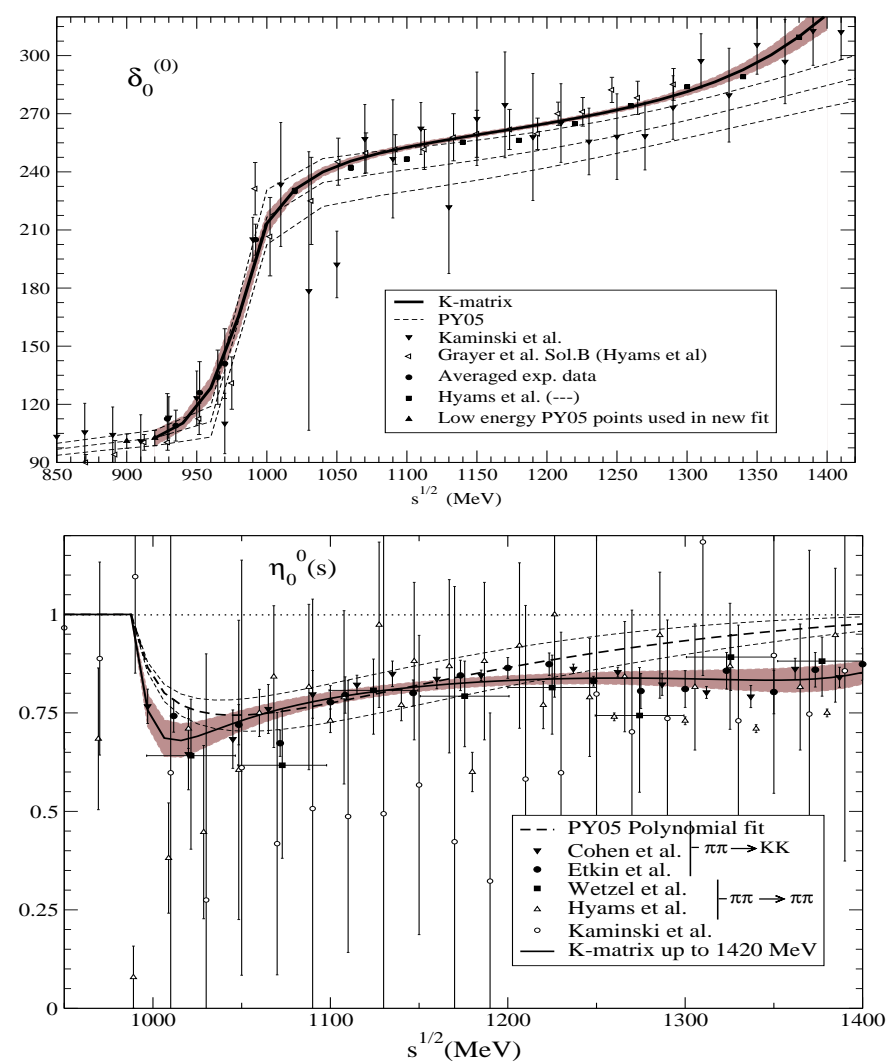

Fig. 1. Phase shifts and inelasticities of the $S 0$ wave fitted using the K-matrix approach of [2] (solid lines). Dotted lines for the results of [1].

the slope parameter calculated from the Froissart-Gribov representation. The resulting $\chi^{2} /$ d.o. $f=0.65$.

The inelasticity is parametrized in the same way as in [1] and fitted to the experimental data of ref. $[3,6,8]$

$$
\eta_{2}^{(0)}(s)=1-\epsilon \frac{k_{2}(s)}{k_{2}\left(M_{f_{2}}^{2}\right)}
$$

Results of the fits for phase shifts and inelasticities are presented in Fig. 2.

\subsection{The $P$ wave}

In the $P$ wave, above the $K \bar{K}$ threshold we have used a more flexible parametrization than in [1]:

$$
\begin{gathered}
\delta_{1}(s)=\lambda_{0}+\sum_{i=1}^{2} \lambda_{i}\left(\sqrt{s / 4 m_{K}^{2}}-1\right)^{i}, \\
\eta_{1}(s)=1-\sum_{i=1}^{2} \epsilon_{i}\left(\sqrt{1-4 m_{K}^{2} / s}\right)^{i},
\end{gathered}
$$

where $\lambda_{0}$ is fixed by the phase shift at $2 m_{K}$ which is obtained from the fit to the pion form factor [11]. We have then fitted data from $[6,8]$ obtaining $\chi^{2} /$ d.o.f. $=0.6$ and $\chi^{2} /$ d.o.f. $=1.1$ for the phase shifts and inelasticity, respectively. The results are presented in Fig. 3. 

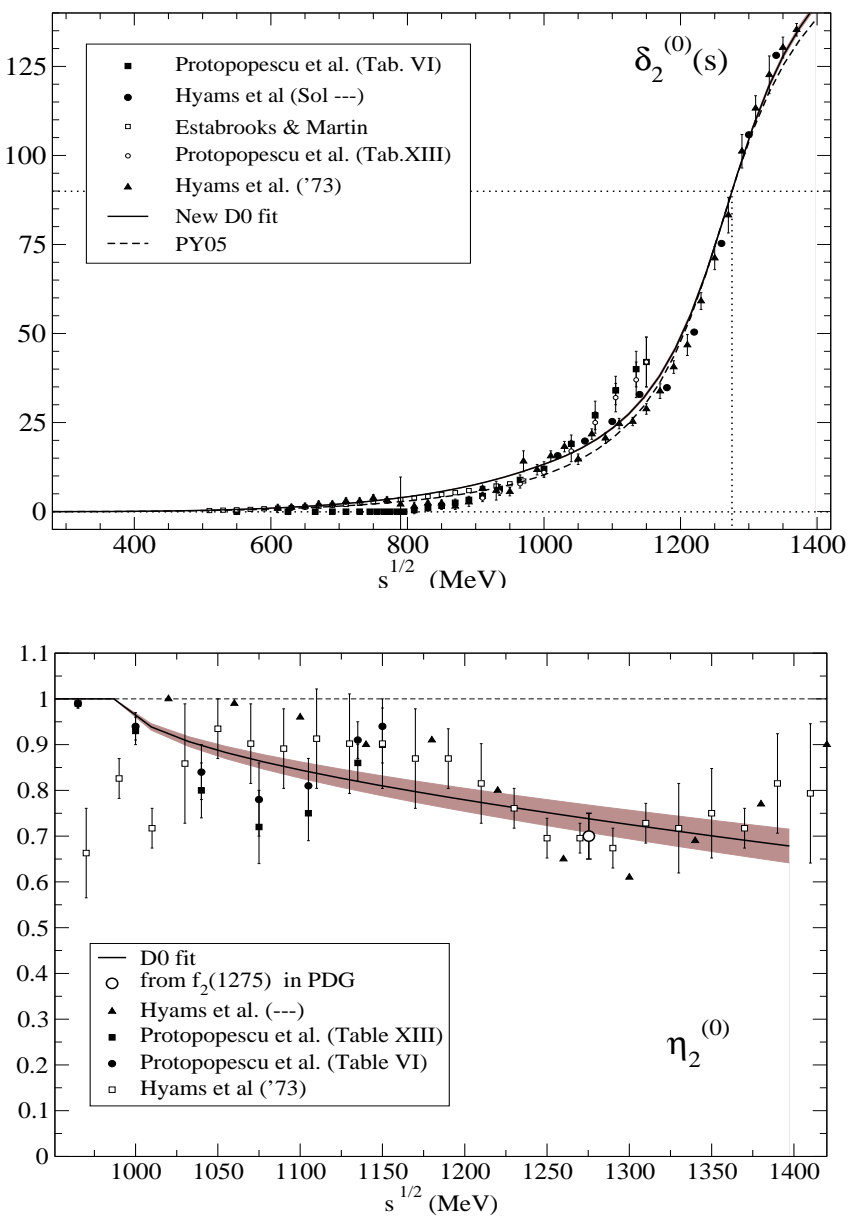

Fig. 2. The $D 0$ wave phase shifts and inelasticities determined in [2] (solid lines) and in [1] (dotted line - only for phase shifts). Dark areas denote the errors, which for the phase shifts have just the thickness of the line.
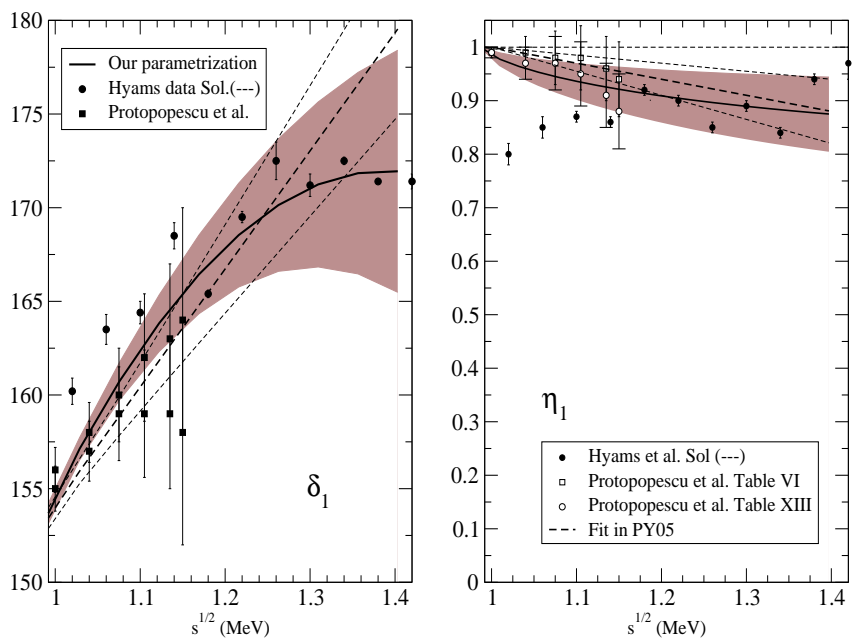

Fig. 3. Fits to the $P$ wave phase shifts and inelasticity (solid lines). Dark areas show the errors of our results. The dotted lines represent results obtained in [1]. $\delta_{2}^{(2)}(\mathrm{s})$

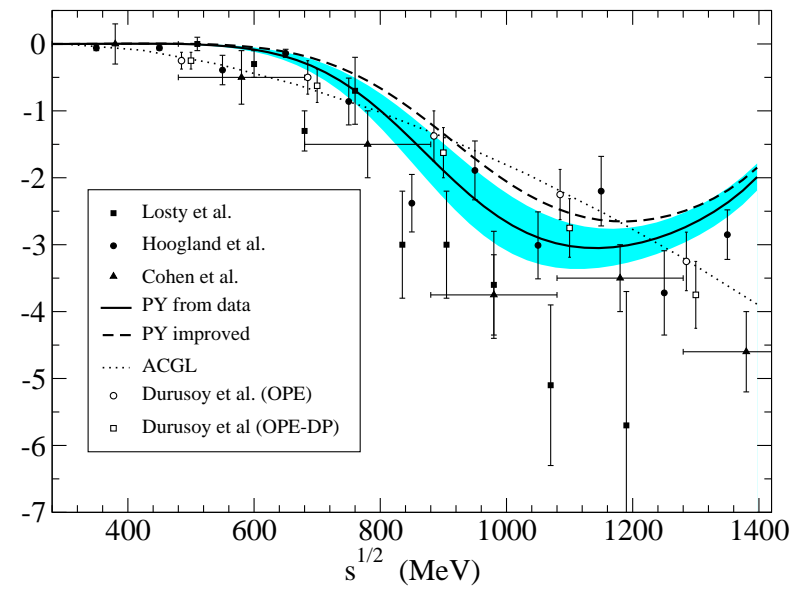

Fig. 4. Results of the fit to the $D 2$ wave. The solid line denotes the fit to the experimental data only and the dashed one the fit to the data and FDR [1]. For the data enclosed in the figure see references in [2].

\subsection{The $D 2$ wave}

In the $D 2$ wave we have used one single parametrization up to $1.42 \mathrm{GeV}$ with four free parameters

$\cot \delta_{2}^{(2)}=\frac{s^{1 / 2}}{2 k^{5}}\left(B_{0}+B_{1} w(s)+B_{1} w(s)^{2}\right) \frac{m_{\pi}^{4} s}{4\left(m_{\pi}^{2}+\Delta^{2}\right)-s}$,

where $\Delta$ fixes zero of the phase shift near the $\pi \pi$ threshold. Since the data on this wave are not accurate we have added one more constrain using the scattering length calculated from the Froissart-Gribov representation [1]. As a result we have obtained the fit presented in Fig. 4.

The lack of experimental data on inelasticity led us to estimate it from a model (see ref. [2]) writing

$$
\eta_{2}^{(2)}=1-\epsilon(1-\hat{s} / s)^{3}
$$

with $\sqrt{\hat{s}}=1.05 \mathrm{GeV}$ and $\epsilon=0.2 \pm 0.2$. The inelasticity is very small and even negligible below $1.25 \mathrm{GeV}$.

\section{Regge parametrization}

In the analysis of $[1,10]$ the fits were made with the assumption of "exact degeneracy" of the intercept parameters $\alpha_{\rho}=\alpha_{P^{\prime}}$ for $\rho$ and $f_{2}$ exchange. In our new approach this degeneracy has been lifted. As a consequence, there was a very small change in the high energy behaviour of scattering amplitudes (especially a little for higher energies) but, as can be seen in next section, even such a small change could be significant given the level of precision achieved in our FDR calculation. The energy dependence of the new scattering amplitudes after eliminating the degeneracy is seen in Fig. 5. 

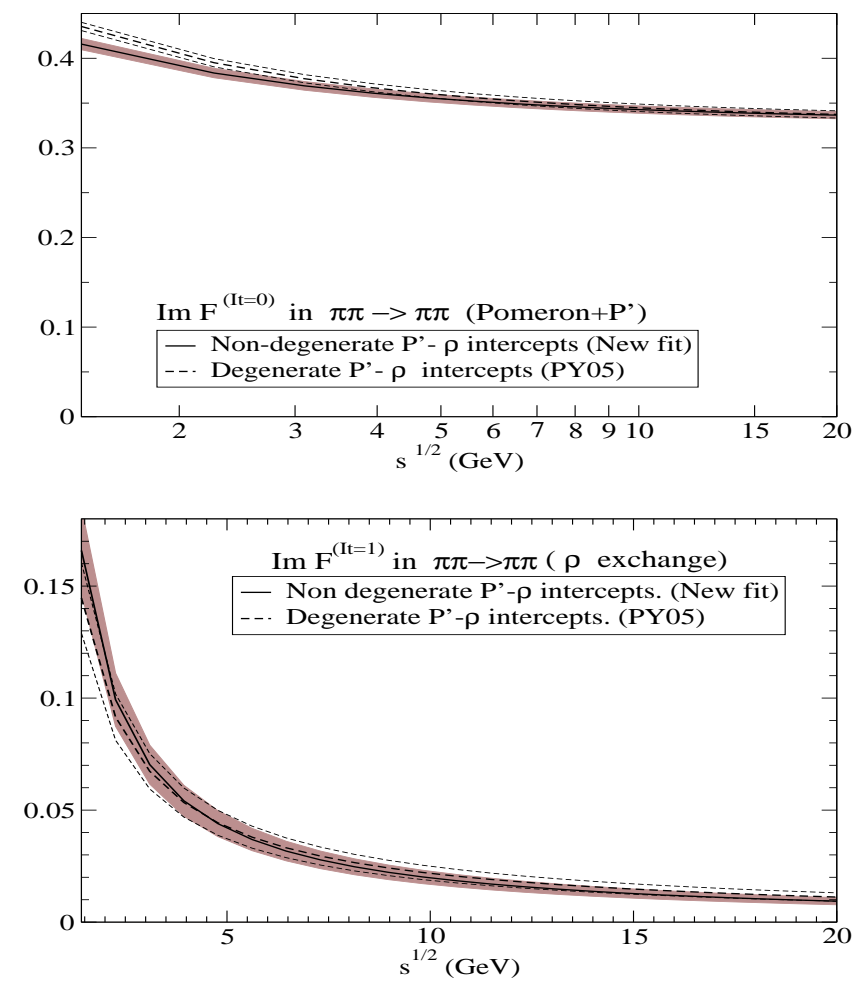

Fig. 5. The scattering amplitudes calculated with "exact degeneracy" (broken lines) and without this condition (solid lines). Dark bands stand for uncertainties.

\section{Implementation of forward dispersion relations}

The $S, P$ and $D$ waves presented in Section 2 together with the improved Regge description in the previous section have been examined in the same way as in [1], by checking the FDR's, but without imposing them as constraints. Thus, in Fig. 6 we present the results from the amplitudes in [1] obtained from fits to data. In contrast, in Fig. 7 we show results using the improved fits given in $[2]$ that we are reviewing here. The $F_{00}, F_{0+}$ and $I_{t}=1$ names used in Figs 6 and 7 correspond to the FDR's for the $\pi^{0} \pi^{0}, \pi^{0} \pi^{+}$and $t$-channel isospin 1 scattering amplitudes, whose full mathematical expressions can be found in [1] and [2]. The word "dispersive" denotes results obtained from the integrals in the FDR's whereas "direct" means the real parts evaluated directly from parametrizations.

We provide in Table 1 the FDR's averaged $\chi^{2}$ obtained over the range from threshold up to $930 \mathrm{MeV}$ or $1420 \mathrm{MeV}$. Note that the modifications in the $S$ and $P$ waves above $K K$ threshold, as well as of the $D$ wave, lead to significant improvement of accuracy in the FDR for the $\pi^{0} \pi^{0}$ scattering amplitudes when compared with the previous results in [1]. The final decrease of the $\chi^{2}$ for $\pi^{0} \pi^{+}$is also due to the influence of the new Regge amplitude. Note that in the $I_{t}=1$ case there is a tiny deterioration despite a significant $\chi^{2}$ decrease due to the Regge part.
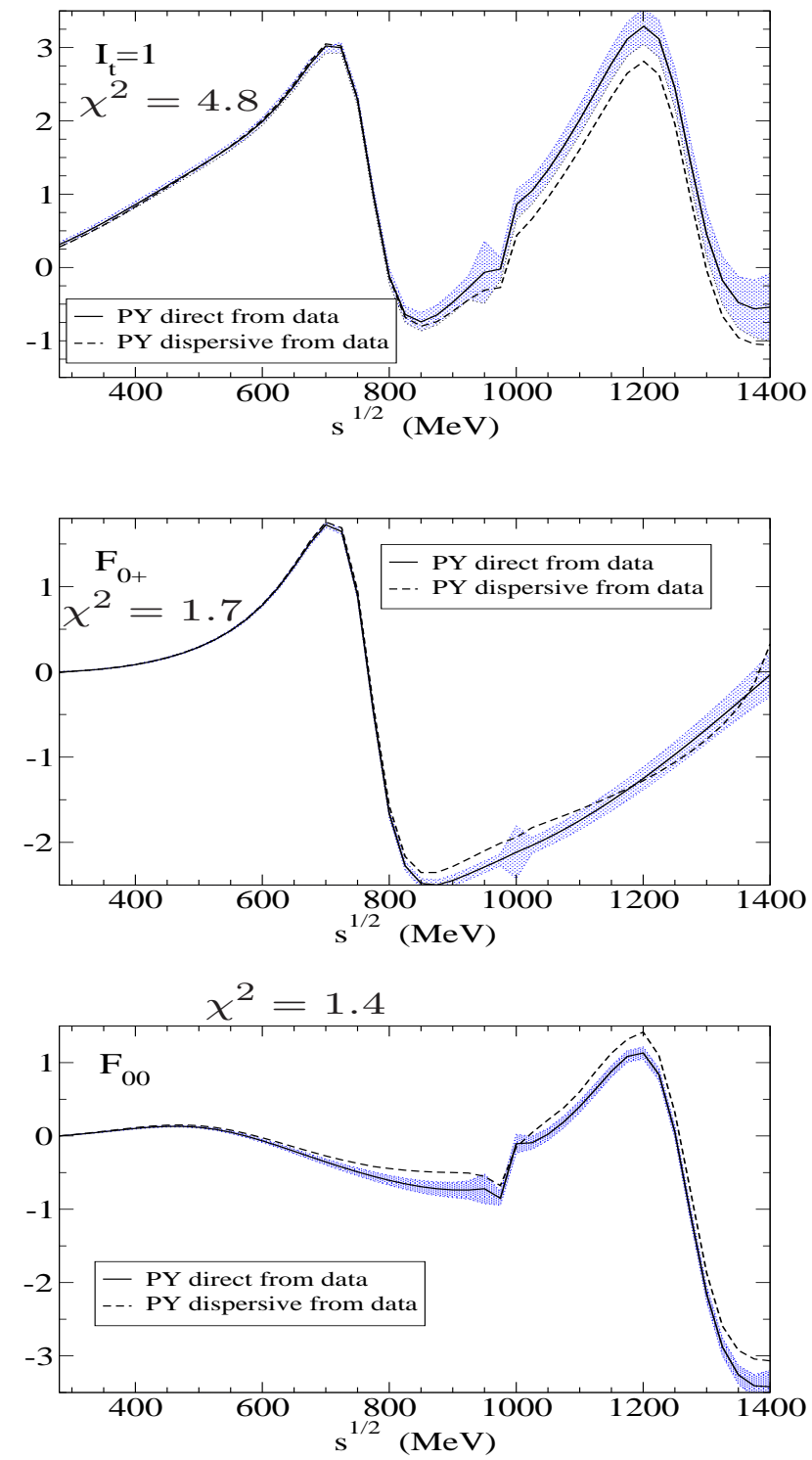

Fig. 6. The $\pi^{0} \pi^{0}, \pi^{0} \pi^{+}$and t-channel 1 forward dispersion relations described in previous analysis [1]. The values of the $\chi^{2}$ denote averaged values over all 25 points chosen in the energy range from the $\pi \pi$ threshold to $1.42 \mathrm{GeV}$.

\section{Tests using Roy's equations}

We here present an advance of our ongoing analisys where we test our new $\pi \pi$ scattering amplitudes using Roy's equations [12-14]:

$$
\begin{aligned}
& \operatorname{Re} f_{\ell}^{I}(s)=a_{0}^{0} \delta_{I 0} \delta_{\ell 0}+a_{0}^{2} \delta_{I 2} \delta_{\ell 0}+ \\
& \left(2 a_{0}^{0}-5 a_{0}^{2}\right)\left(\delta_{I 0} \delta_{\ell 0}+\frac{1}{6} \delta_{I 1} \delta_{\ell 1}-\frac{1}{2} \delta_{I 2} \delta_{\ell 0}\right) \frac{s-4 \mu^{2}}{12 \mu^{2}}+ \\
& \sum_{I^{\prime}=0}^{2} \sum_{\ell^{\prime}=0}^{1} f_{4 \mu^{2}}^{s_{\max }} d s^{\prime} K_{\ell \ell^{\prime}}^{I I^{\prime}}\left(s, s^{\prime}\right) \operatorname{Im} f_{\ell^{\prime}}^{I^{\prime}}\left(s^{\prime}\right)+d_{\ell}^{I}\left(s, s_{\max }\right),
\end{aligned}
$$


Table 1. Comparison of averaged $\chi^{2}$ for different FDR obtained in previous analysis [1] and in presented one (new $\delta, \eta$ and new Regge) in two energy ranges. Numbers correspond to fits to experimental data only (without constraints from FDR).

\begin{tabular}{cccc} 
results of $[1]$ & new $\delta, \eta$ & new Regge & Energy range \\
\hline for $\pi^{0} \pi^{0}$ & dispersion relations: & \\
\hline 3.8 & 1.52 & 1.41 & $s^{1 / 2}<930 \mathrm{MeV}$ \\
4.8 & 1.76 & 1.63 & $s^{1 / 2}<1420 \mathrm{MeV}$ \\
\hline for $\pi^{0} \pi^{+}$ & dispersion relations: & \\
1.7 & 1.75 & 1.60 & $s^{1 / 2}<930 \mathrm{MeV}$ \\
1.7 & 1.60 & 1.44 & $s^{1 / 2}<1420 \mathrm{MeV}$
\end{tabular}

for $I_{t}=1$ scattering amplitudes:

$\begin{array}{lllc}0.2 & 0.57 & 0.32 & s^{1 / 2}<930 \mathrm{MeV} \\ 1.4 & 2.32 & 1.76 & s^{1 / 2}<1420 \mathrm{MeV}\end{array}$
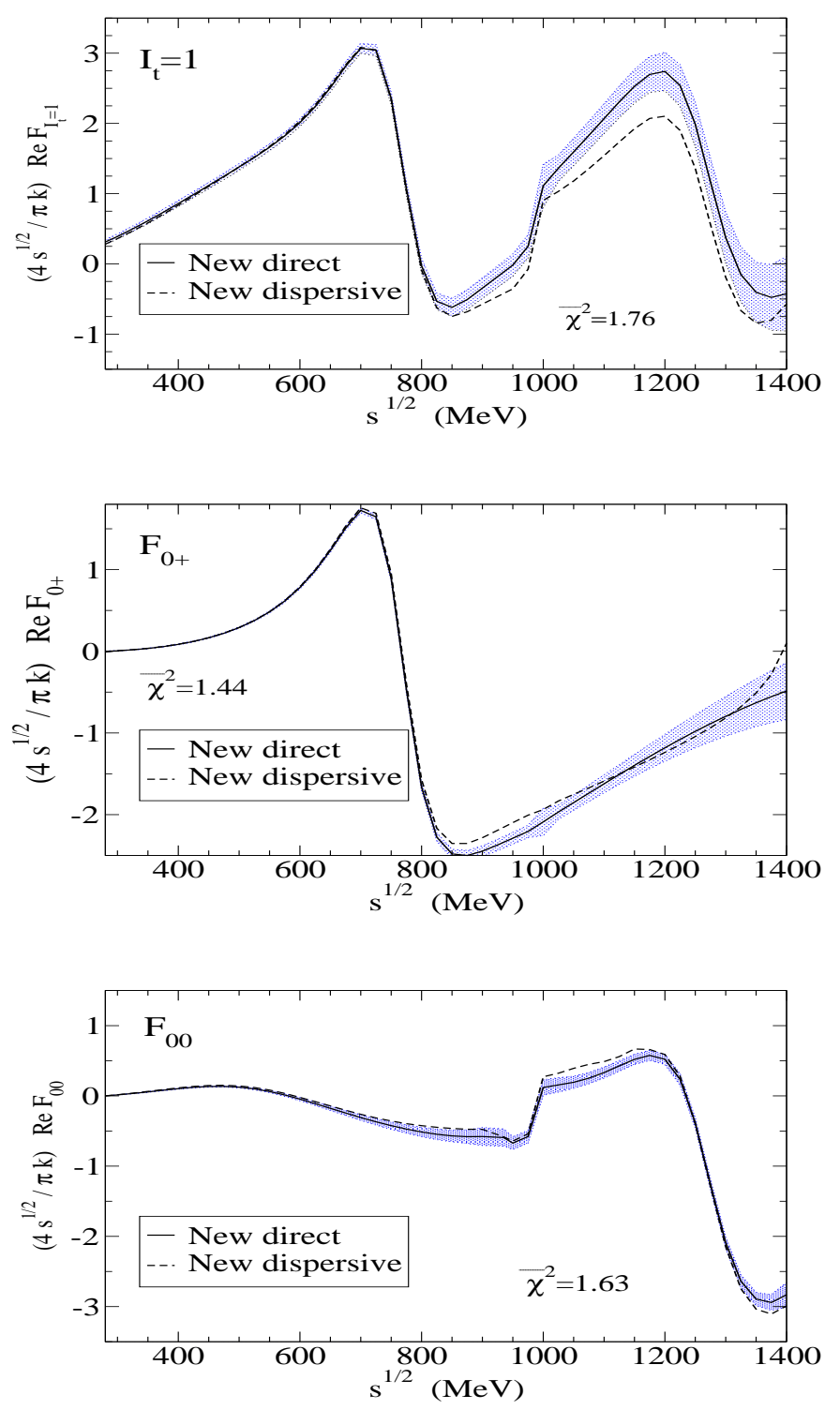

Fig. 7. Dispersion relations for new $S, P$ and $D$ waves described in this paper. The $\chi^{2}$ definition as in Fig. 6 where

$$
f_{\ell}^{I}(s)=\sqrt{\frac{s}{s-4 \mu^{2}}} \frac{1}{2 i}\left(\eta_{\ell}^{I} e^{2 i \delta_{\ell}^{I}}-1\right),
$$

with $a_{0}^{0}$ and $a_{0}^{2}$ being the $S 0$ and $S 2$ scattering lengths, $K_{\ell \ell^{\prime}}^{I I^{\prime}}\left(s, s^{\prime}\right)$ known kernels and $d_{\ell}^{I}\left(s, s_{\max }\right)$ the so called driving terms. In our calculations we have chosen $s_{\max }=$ $103 m_{\pi}^{2}$.

In Fig. 8 we show the real part of the $S 0, S 2$ and $P$ partial waves obtained from Eq.(8), (continuous line, called Roy ${ }^{\text {out }}$ ) versus the real part obtained directly from our parametrizations, (dashed line, called called Roy ${ }^{i n}$ ).

The agreement is remarkable, taking into account the uncertainties (the dark areas in Fig. 8). Furthermore, the agreement is even more impressive, taking into account that we have not imposed any constraints from FDR or Roy's equations themselves and that the amplitudes come just from fits to data (that is why they are labeled "from data" in the Figure). Moreover, we use the new $S, P$ and $D$ waves described in Section 2 and the Regge model with different intercepts $\alpha_{\rho}(0)$ and $\alpha_{P^{\prime}}(0)$, and all of them have experimental errors even smaller than those of [1].

\section{Conclusions}

The results reviewed here indicate that the improvement in the fits to data in the $S$ and $P$ waves above $K K$ threshold and the $D$ wave described in Section 2 together with a slight improvement in Regge trajectories, allowing for non $\rho-f$ degeneracy, also improves the fulfillment of forward dispersion relations. Despite the smaller errors of those amplitudes, the averaged $\chi^{2}$ is indeed lower than in previous analysis [1]. We have shown that those amplitudes fulfill also quite well Roy's equations, and therefore, crossing symmetry, up to roughly $1 \mathrm{GeV}$.

Following, however, the analysis done in [1] one can think about a wider implementation of FDR including them into the fits together with the already fitted experimental data. We report briefly on our progress in this approach, where, for the moment, we allow for a variation of all the amplitude parameters except, the $P$ wave above $K K$ threshold and just the $\alpha$ and $\beta$ parameters in the $\mathbf{K}$-matrix. Although our results are just preliminary, we already noticed significant decreases of the averaged $\chi^{2}$ for all three FDR. The preliminary values for $F_{00}$ decreased from 1.63 to 0.42 , for $F_{0+}$ changed slightly from 1.44 to 1.48 and for $I_{t}=1$ decreased from 1.76 to 0.89 . The more spectacular improvement, however, has been noticed in the Roy equations. Preliminary averaged $\chi^{2}$ values decreased from 1.03 to 0.47 for the $S 0$ wave, from 1.18 to 0.53 for the $S 2$ wave and from 0.65 to 0.02 for the $P$ wave. This improvement can be clearly seen when comparing Figs 8 and 9. Imposing the constrains from Roy equations and particularly from FDR, which is much stringent, leads to modifications in the $S, P$ and $D$ waves by less than $1 \sigma$ (with the exception for $D 2$ wave where the empirical fit changed by $\sim 1.3 \sigma$ ) and to negligible modifications in all other waves. The resulting uncertainties are also significantly reduced with this approach as can be seen, just for 

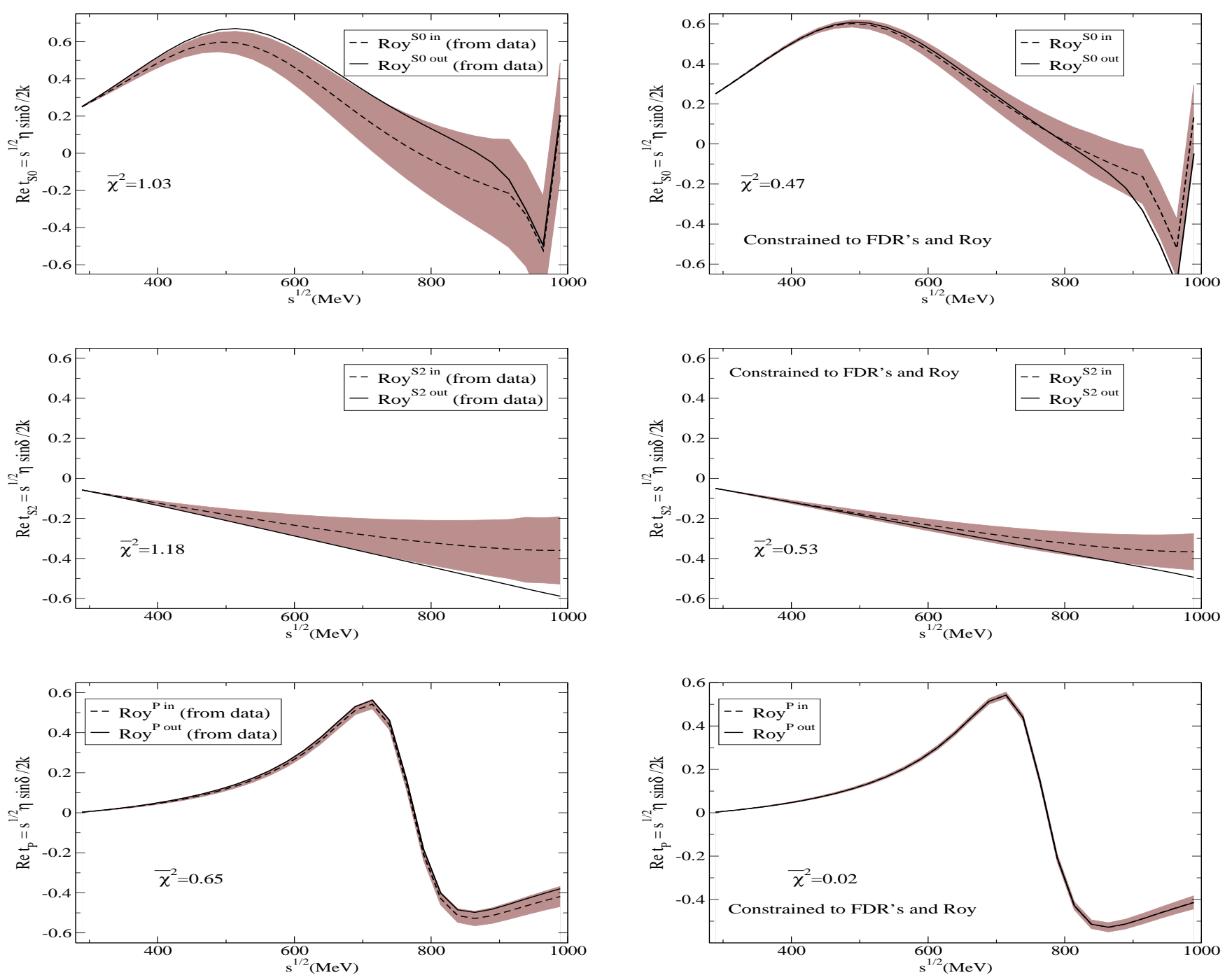

Fig. 8. Differences between real parts of amplitudes calculated directly from amplitudes and those from the integral representation of Roy's equations. The notation is explained in the Section 5 .

Roy equations, in Figs 8 and 9. At present we are finishing the determination of the final parameters and their uncertainties.

\section{References}

1. J. R. Peláez, F. J. Ynduráin, Phys. Rev. D71, (2005) 074016.

2. R. Kamiński,J. R. Peláez, F. J. Ynduráin, Phys. Rev. D74, (2006) 014001 and Erratum Phys. Rev. D74 (2006) 079903.

3. B. Hyams, et al., Nucl. Phys. B64, (1073) 134. Estabrooks, P., and Martin, A. D., Nucl. Physics, B79, (1974) 301.

4. G. Grayer, et al., Nucl. Phys. B75, (1974) 189.

Fig. 9. As in Fig. 8 but for data fits constrained by FDR and Roy's equations.

5. R. Kamiński, L. Leśniak, K. Rybicki, Z. Phys. C74, (1997) 79 (1997) and Eur. Phys. J. direct C4, (2002) 4.

6. B. Hyams, et al., Nucl. Phys. B100, (1975) 205.

7. $\pi \pi \rightarrow \bar{K} K$ scattering: W. Wetzel et al., Nucl. Phys. B115, (1976) 208; D. Cohen et al., Phys. Rev. D22, (1980) 2595; E. Etkin et al., Phys. Rev. D25, (1982) 1786.

8. S. D. Protopopescu, et al., Phys Rev. D7, (1973) 1279.

9. Eidelman, S., et al., Phys. Letters B592, 1 (2004).

10. J. R. Pelaez and F. J. Yndurain, Phys. Rev. D 69, 114001 (2004).

11. J. F. de Trocóniz, F. J. Ynduráin, Phys Rev. D65, (2002) 093001; Phys Rev. D71, (2005) 073008.

12. S. M. Roy, Phys. Lett. B 36, 353 (1971).

13. B. Ananthanarayan, G. Colangelo, J. Gasser and H. Leutwyler, Phys. Rept. 353, 207 (2001).

14. R. Kamiński, L. Leśniak, B. Loiseau, Phys. Lett. B 551, (2003) 241. 
